Pacific Journal of Mathematics

ASYMPTOTIC APPROACH TO PERIODIC ORBITS AND
LOCAL PROLONGATIONS OF MAPS 


\title{
ASYMPTOTIC APPROACH TO PERIODIC ORBITS AND LOCAL PROLONGATIONS OF MAPS
}

\author{
ROBERT J. SACKER
}

\begin{abstract}
This paper is concerned with a flow on a metric space, and some topological properties of the set of orbits which are asymptotic to a given invariant subset, with particular emphasis on the flow near an invariant Jordan curve (e.g., a periodic orbit) in an orientable $n$-manifold $M^{n}$. The investigation began with the asking of the simple question: Can a periodic orbit $J$ of a vector field in $R^{n}$ be the $\omega$-limit set of precisely one orbit distinct from $J$ ? It is shown that if the periodic orbit $J$ is a maximal element in the class of invariant continuua lying in a neighborhood of $J$, then the answer is negative and in fact the set of orbits asymptotic to $J$ as $t \rightarrow \infty$ has some of the same topological properties already known from the stable manifold theorems in the case of an elementary periodic orbit of a flow generated by a smooth ordinary differential equation.
\end{abstract}

The assumption of maximality of $J$ is replaced by an even weaker condition and the notions of a local quasi-section and its associated mapping are introduced to handle the case in which $J$ is an invariant Jordan curve. For such a mapping a local prolongation is defined which proves useful in studying properties of the orbits asymptotic to $J$.

Let $\pi: M^{n} \times R \rightarrow M^{n}$ be a flow, i.e., $\pi(x, 0)=x$ for all $x \in M^{n}$, $\pi(\pi(x, s), t)=\pi(x, s+t)$ and $\pi$ is continuous. Denote $\pi(x, t)$ by $x \cdot t$ and for $E \subset M^{n}$ and $A \subset R, E \cdot A=\bigcup\{x \cdot t: x \in E, t \in A\}$. Our initial approach in the case $J$ is a periodic orbit is to consider the induced map $T$ of a surface of section $\Sigma$ at a point $J_{0}$ in $J, J_{0}$ thus being fixed under $T$. In $\S 3$ we introduce the notion of local prolongation of a mapping in metric space and as a special case it turns out that if there is a $\Sigma$-neighborhood $U$ of $J_{0}$ such that $J_{0}$ is maximal in $U$ then there is a continuum $\Gamma \subset \Sigma$ lying in $U$ and extending from $J_{0}$ to the boundary of $U$ such that for all $x \in \Gamma, T^{n} x \rightarrow J_{0}$ as $n \rightarrow \infty$. By maximal we mean that there is no continuum $K$ with $J_{0} \in K \subset U$ which is invariant under $T$, i.e., $T K=K$, except for the obvious choice $K=J_{0}$. Thus in the maximal case the above question is answered in the negative. With the condition of being maximal dropped we construct an example ( $\$ 3$ ) of a map $T: R^{2} \rightarrow R^{2}$ leaving the origin fixed and such that if $\Delta$ is the closed unit disc, then the subset $F=\left\{x \in \Delta-(0,0): T^{n} x \rightarrow(0,0)\right.$ as $\left.n \rightarrow \infty\right\}$ is discrete, $F=\left\{x_{1}\right.$, $\left.x_{2}, \cdots\right\}$ with $T x_{n}=x_{n+1}$. Thus, if we suspend this mapping to a 
continuous flow we obtain a periodic orbit $J$ having precisely one orbit (distinct from $J$ ) which is positively asymptotic to $J$.

Let $X$ be a solid torus neighborhood of the periodic orbit $J$ in $M^{n}$ and define $A^{+}=\{x \in X: x \cdot[0, \infty) \subset X$ and $\omega(x) \subset J\}$. We assume that there is an $x \in X-J$ such that $\omega(x) \subset J$, i.e., $A^{+}-J \neq \varnothing$. If $J$ is assumed to be a maximal element in the class of continua in $X$ which are $\pi$-invariant, then there is a neighborhood $U$ in $\Sigma$ such that $J_{0}$ is maximal in $U$ and it is easily seen that $\Gamma$ will then lie in $A^{+}$. More can be said however about $A^{+}$. In fact it will be shown in $\$ 5$ that there is an open solid torus neighborhood $V$ of $J$ in $M^{n}$ with $\bar{V} \subset$ int $X$ and such that $(X-V) \cap A^{+}$carries a Čech 1-cycle which is nonbounding in $X$ and homologous over $A^{+}$to the basic 1-cycle carried by $J$. This is a generalization of the classical case in which one assumes that $\pi$ is generated by a sufficiently smooth vector field in a smooth $M^{n}$ and $J$ is a periodic orbit with $n-1$ of its Floquet exponents having nonzero real parts. In that case it follows [8] that for appropriately chosen $X, A^{+}$is a smooth manifold with boundary. For example taking $n=3$, it is easy to show that $A^{+}$is either equal to $X$, an annulus or a möbius band and therefore $(X-V) \cap A^{+}$, for an appropriate open torus neighborhood $V$ of $J$ satisfying $\bar{V} \subset \operatorname{int} X$, is either equal to $X-V$, a pair of disjoint annuli or a single annulus. The existence of the continuum $\Gamma$ and the Cech 1-cycle then follow by inspection.

Returning to the general case, it turns out that the foregoing conclusions depend on the behavior of the flow near $J$ and not in $J$ itself. For this purpose we introduce the notion of a local quasisection for an invariant set and show that if $J$ is an invariant Jordan curve which admits a local quasi-section $\Sigma$ then under certain additional conditions the existence of the continuum $\Gamma \subset \Sigma$ and the 1cycle in $(X-V) \cap A^{+}$are guaranteed. In what follows we even drop the condition that $J$ be maximal and replace it with much weaker conditions.

Announcement of some of these results appeared in [9]. See also Conley [3], and Churchill [2] for related results concerning invariant sets which carry cohomology, a concept introduced in [3].

2. Local quasi-sections. The following generalizes the notion of a surface of section for a periodic orbit. Although the existence of a local quasi-section is not explicitly assumed in the remainder of the paper, its existence (together with the associated map) is sufficient to verify the assumption called Condition $\mathrm{A}$ in $\S 5$ (see Remark 2 following Condition A).

Definition. Let $W$ be a metric space, $\pi: W \times R \rightarrow W$ a flow and 
$I \subset W$ a compact $\pi$-invariant subset. Then $I$ is said to admit local quasi-section $\Sigma$ if there exists a compact subset $\Sigma \subset W$ satisfying the following: (1) There exists an open neighborhood $N$ of $I$ such that if $N^{\prime} \subset N$ is any open neighborhood of $I$ then there exists an open neighborhood $N^{\prime \prime} \subset N^{\prime}$ of $I$ and functions $\tau^{+}: N^{\prime \prime}-I \rightarrow(0, \infty)$ and $\tau^{-}: N^{\prime \prime}-I \rightarrow(-\infty, 0)$ such that for all $x \in N^{\prime \prime}-I, x \cdot\left(0, \tau^{+}(x)\right) \subset$ $N^{\prime}-\Sigma$ and $x \cdot\left(\tau^{-}(x), 0\right) \subset N^{\prime}-\Sigma$ while $x \cdot \tau^{+}(x)$ and $x \cdot \tau^{-}(x) \in \Sigma \cap N^{\prime}$. (2) For each $x \in(N-I) \cap \Sigma$ and $\varepsilon>0$ there exists $\delta, 0<\delta<\varepsilon$ and an open $W$-neighborhood $B$ of $x$ such that

(a) for all $x^{\prime} \in B$ there exists a $t \in(-\delta, \delta)$ such that $x^{\prime} \cdot t \in \Sigma$ and

(b) if $\xi=x^{\prime} \cdot t$ then $\{\xi \cdot[-\delta, 0) \cup \xi \cdot(0, \delta]\} \cap \Sigma=\varnothing$.

Remarks. (1) In the definition let $N^{\prime}=N$ initially and let $N_{0}^{\prime \prime}$ be the neighborhood guaranteed by (1) and $\tau_{0}^{+}: N_{0}^{\prime \prime}-I \rightarrow(0, \infty)$ the corresponding function. Then it is easy to see that for any other $N^{\prime}$ the corresponding $N^{\prime \prime}$ and $\tau^{+}$satisfy $\tau^{+}=\tau_{0}^{+} \mid N^{\prime \prime}-I$. Similarly for $\tau^{-}$.

(2) $\tau_{0}^{+}$and $\tau_{0}^{-}$restricted to $N_{0}^{\prime \prime} \cap \Sigma$ are continuous.

DEFINITION. If $\Sigma$ is a local quasi-section for $I$ we define the associated mapping $T$ as follows: Let $A=\Sigma \cap I$ and $U=N_{0}^{\prime \prime} \cap \Sigma$. Then define $T$ and $T^{-1}: U-A \rightarrow \Sigma$ by $T(x)=x \cdot \tau_{0}^{+}(x), T^{-1}(x)=x \cdot \tau_{0}^{-}(x)$. Clearly $T^{-1}$ is the inverse of $T$, each is continuous and $T^{ \pm 1}(x) \rightarrow A$ as $x \rightarrow A$. $T$ represents the map which carries a point $x$ to the point $T(x)$ at which the positive semiorbit through $x$ next strikes $\Sigma$.

If $I$ is a periodic orbit and $W$ locally compact then there exists a compact local section at any point $p \in I,[1, \mathrm{p} .50],[7]$ and $\Sigma$ is afortiori a local quasi-section with $A=p$. In this case $T$ is even defined as a continuous function at $A$ leaving $A$ fixed. Even if we modify the flow by placing stationary points on $I$, leaving orbits in $W-I$ intact, $\Sigma$ is still a local quasi-section and $T$ is still extendible to $A$ leaving $A$ fixed. However, in general, for a local quasi-section, $T$ need not even be defined on $A$ nor extendible to $A$ as a continuous mapping, e.g., let $I$ be a 2-torus in $R^{3}$ with periodic coordinates $0 \leqq$ $x_{1}, x_{2} \leqq 2 \pi$ and $y$ the normal coordinate, $|y| \leqq \delta$. Let $\Sigma=\left\{\left(x_{1}, x_{2}, y\right)\right.$ : $\left.x_{1}=0,0 \leqq x_{2} \leqq 2 \pi,|y| \leqq \delta\right\}$ and $A$ the circle on $I$ defined by $x_{1}=0$. For the differential system

$$
\begin{aligned}
\dot{x}_{1} & =\left|\sin \frac{x_{1}}{2}\right|+|y| \\
\dot{x}_{2} & =1 \\
\dot{y} & =-y
\end{aligned}
$$


it is easily seen that for $p \in \Sigma-A$ and $p_{0} \in A, \varlimsup_{p \rightarrow p_{0}} T(p)=A$.

3. Local prolongation of a mapping. In this section we introduce the notion of a local prolongation which, roughly speaking, accomplishes the following: Taking a simple situation, let $A$ be a fixed-point of a homeomorphism $T$ defined in a neighborhood $Y$ of $A$. We wish to associate with $A$ a closed subset $P^{+} \subset Y-A$ which abuts on $A$ and is positively invariant, $T P^{+} \subset P^{+}$. Under certain conditions the set $P^{+} \cup A$ will then generalize the classical local stable manifold of $A$. Only the most basic properties of local prolongations suitable to the problem at hand will be established here. The local prolongation introduced here is similar to the first prolongation for a continuous flow introduced by T. Ura and discussed in [1].

Let $\Sigma$ be a compact metric space and $A \subset \Sigma, A \neq \varnothing$, a closed subset. Let $Y \subset \Sigma$ be the closure of an open neighborhood of $A$ and define $Y^{\prime}=Y-A$ and $\Sigma^{\prime}=\Sigma-A$. Suppose $T$ is a mapping such that $Y^{\prime} \subset$ Domain $T \cap$ Domain $T^{-1}$ and $T: Y^{\prime} \rightarrow T\left(Y^{\prime}\right)$ and $T^{-1}: Y^{\prime} \rightarrow$ $T^{-1}\left(Y^{\prime}\right)$ are homeomorphisms. Further assume that $T$ (and $T^{-1}$ ) satisfies the condition: For every $\Sigma$-open neighborhood $U$ of $A$ there exists a $\Sigma$-open neighborhood $V$ of $A$ such that $T\left(V \cap Y^{\prime}\right) \subset U \cap Y^{\prime}$. Note that $T$ need not even be defined on $A$. However, if $T$ is a homeomorphism defined on a $\Sigma$-neighborhood of $A$ with $T A=A$ then the above conditions are automatically satisfied.

Of particular interest is the case in which $\Sigma$ is a local quasi-section for an invariant set $I, A=\Sigma \cap I$ and $T$ is the associated mapping.

For $P \subset Q$ we denote the relative boundary of $P$ in $Q$ by $\partial_{Q} P=$ $\bar{P} \cap \overline{Q-P}$ and the relative interior int ${ }_{Q} P=P-\partial_{Q} P$. Let Comp $F$ denote the collection of components of $F$.

Definition. A set $E$ is said to dominate the set $A$, written $E \gg$ $A$ if $E \supset A$ and for each $E_{0} \in \operatorname{Comp} E$ there exists $A_{0} \in \operatorname{Comp} A$ such that $E_{0} \supset A_{0}$.

We mention some elementary properties for arbitrary subsets $E, F, G, A_{\alpha}$ of the space $Y$ :

(3.1) $E \gg E$; if $E \gg F$ and $F \gg E$ then $E=F$; if $E \gg F$ and $F \gg G$ then $E \gg G$.

(3.2) If $A_{\alpha} \gg A$ for all $\alpha \in \mathscr{A}$, an arbitrary index set, then $\mathrm{U}_{\alpha \in \mathscr{A}} A_{\alpha} \gg A$.

(3.3) If $E \gg A$ then $\bar{E} \gg A$.

(3.4) Let $\left\{A_{\alpha} \subset Y, \alpha \in \mathscr{A}\right\}$ be a collection of closed subsets of a a compact $Y$ where $\mathscr{A}$ is totally ordered and $\alpha<\beta$ implies $A_{\alpha} \subset A_{\beta}$. If for some closed subset $A \subset Y, A_{\alpha} \gg A$ for all $\alpha \in \mathscr{A}$ then

$$
\bigcap_{\alpha \in \mathscr{A}} A_{\alpha} \gg A \text {. }
$$


(3.5) If follows from the above that if $\mathscr{A}$ is totally ordered and $\left\{E_{\alpha} \subset Y^{\prime}, \alpha \in \mathscr{A}\right\}$ is an arbitrary collection of subsets such that $E_{\alpha} \cup A \gg A$ for all $\alpha \in \mathscr{A}$ then $\left(\overline{\lim }_{\alpha \in \rightsquigarrow} E_{\alpha}\right) \cup A \gg A$ where

$$
\varlimsup_{\alpha \in \mathscr{N}} E_{\alpha}=\bigcap_{\alpha \in \mathcal{N}} \overline{\bigcup_{\beta<\alpha} E_{\beta}} \text {. }
$$

(3.6) If $E \subset Y$ is connected and $E \cap A \neq \varnothing$ then $E \cup A \gg A$. We state without proof the following

Lemma 3.7. Let $F \subset Y^{\prime}$ be such that $T^{-1} F \subset Y^{\prime}$ and $F \cup A \gg A$. Then $\left(T^{-1} F\right) \cup A \gg A$. A similar statement holds for $T$.

We next define certain positively and negatively invariant subsets of $Y^{\prime}$. Let

$$
\begin{aligned}
I^{+}\left(Y^{\prime}\right) & =\left\{F \subset Y^{\prime}: F \text { is closed, } F \cup A \gg A, T F \subset F\right\} \\
I^{-}\left(Y^{\prime}\right) & =\left\{F \subset Y^{\prime}: F \text { is closed, } F \cup A \gg A, T^{-1} F \subset F\right\} \\
I\left(Y^{\prime}\right) & =I^{+}\left(Y^{\prime}\right) \cap I^{-}\left(Y^{\prime}\right) .
\end{aligned}
$$

Definition. For an arbitrary $E \subset \Sigma^{\prime}$ define $E \bigcap_{A} Y^{\prime}$ to be the maximal subset $E^{0}$ of $E \cap Y^{\prime}$ having the property that $E^{0} \cup A \gg A$.

We next define a prolongation operator $P^{+}: I^{+}\left(Y^{\prime}\right) \rightarrow I^{+}\left(Y^{\prime}\right)$. Let $F \in I^{+}\left(Y^{\prime}\right)$ and let $\{G(\alpha) \subset Y: 0<\alpha<1\}$ be a collection of open subsets such that $\bigcap_{0<\alpha<1} G(\alpha)=F \cup A$ and if $\alpha<\beta$ then $G(\alpha) \subset G(\beta)$ and there exists $\gamma \in(0, \alpha)$ such that $\overline{G(\gamma)} \subset G(\beta)$. In the relative topology of $Y^{\prime}$ we then have, for $G^{\prime}(\alpha)=G(\alpha) \cap Y^{\prime}, \overline{G^{\prime}(\gamma)} \subset G(\beta)$ for such a $\gamma$ and $\varlimsup_{\lim _{\alpha \in(0,1)}} G^{\prime}(\alpha)=F$. For each $\alpha \in(0,1)$ we define a sequence of subsets of $Y^{\prime}$ by letting $G_{0}(\alpha)=G^{\prime}(\alpha) \bigcap_{A} Y^{\prime}$ and

$$
G_{n+1}(\alpha)=\left[T^{-1} G_{n}(\alpha)\right] \bigcap_{A} Y^{\prime} .
$$

Then define

$$
P_{c}^{+}(F)=\bigcup_{n=0}^{\infty} G_{n}(\alpha)
$$

and

$$
P^{+}(F)=\varlimsup_{\alpha \in(0,1)} P_{\alpha}^{+}(F)
$$

THEOREM 3.8 .

(1) $P^{+}$is a mapping from $I^{+}\left(Y^{\prime}\right)$ to $I^{+}\left(Y^{\prime}\right)$ and for $F \subset \hat{F}$, both in $I^{+}\left(Y^{\prime}\right), F \subset P^{+}\left(F^{\prime}\right) \subset P^{+}(\hat{F})$.

(2a) If for some $F \in I^{+}\left(Y^{\prime}\right)$ and some $\nu \in(0,1)$ it is true that $T^{-n} G^{\prime}(\nu) \subset Y^{\prime}$ for all integers $n \geqq 0$ then $P^{+}(F) \in I\left(Y^{\prime}\right)$ and there exists an $F^{*} \in I^{-}\left(Y^{\prime}\right)$ and a subset $G \subset Y^{\prime}$ such that $F \subset G \subset \bar{G}=F^{*}$.

(2b) In particular if $P^{+}(F) \cap \partial_{\Sigma^{\prime}} Y^{\prime}=\varnothing$ then there exists such 
$a \nu$ and in addition $F^{*} \subset \operatorname{int}_{\Sigma^{\prime}} Y^{\prime}$.

(2c) Further, if $Y$ is locally connected at each point of $F \cup A$ then the subset $G$ may be chosen to be open in $Y^{\prime}$ and such that $G \cup A$ is open in $Y$.

Proof. (1) Clearly $P^{+}$is well defined, i.e., it does not depend on a particular choice of $G(\alpha)$. It is also clear that for

$$
F \subset \hat{F}, P^{+}(F) \subset P^{+}(\hat{F}) .
$$

For all $\alpha, G_{0}(\alpha) \cup A \gg A$ and since $G^{\prime}(\alpha) \supset F$ and $F \cup A \gg A$ we have $G_{0}(\alpha) \supset F$. Since $F \in I^{+}\left(Y^{\prime}\right), T^{-1} G_{0}(\alpha) \supset T^{-1} F \supset F$ and therefore $G_{1}(\alpha) \cup$ $A \gg A$ and $G_{1}(\alpha) \supset F$. Continuing inductively we see that for all $n \geqq 0$, $G_{n}(\alpha) \cup A \gg A$ and $G_{n}(\alpha) \supset F$. Using the properties of "》" mentioned above we see that $P_{\alpha}^{+}(F) \cup A \gg A$ and $P_{\alpha}^{+}(F) \supset F$ and finally $P^{+}(F) \cup$ $A \gg A$ and $P^{+}(F) \supset F$. To finish the proof of (1) we show that $T P \subset P$ where $P=P^{+}(F)$. Let $x \in P$. If $x \in F$ then $T(x) \in F \subset P$ and we are done. If $x \in P-F$ then there exists $\hat{\alpha} \in(0,1)$ such that for all $\alpha \in$ $(0, \hat{\alpha}), x \notin \overline{G_{0}(\alpha)}$. But $x \in P$ implies $x \in \overline{P_{\alpha}^{+}(F)}=\overline{\bigcup_{n=0}^{\infty} G_{n}(\alpha)}$ for all $\alpha \in$ $(0,1)$. Thus $x \in \overline{\mathrm{U}_{n=1}^{\infty} G_{n}(\alpha)}$ for all $\alpha \in(0, \hat{\alpha})$. Thus for each fixed $\alpha \in(0$, $\hat{\alpha})$ there exists a sequence $x_{k} \rightarrow x, x_{k} \in G_{n_{k}}(\alpha)$ for some sequence of integers $n_{k} \geqq 1$. But then $T\left(x_{k}\right) \rightarrow T(x)$ and $T\left(x_{k}\right) \in G_{n_{k-1}}(\alpha)$ and therefore $T(x) \in \overline{P_{\alpha}^{+}(F)}$. But $\alpha \leqq \beta$ implies $\overline{P_{\alpha}^{+}(F)} \subset \overline{P_{\beta}^{+}(F)}$ and therefore $T(x) \in \overline{P_{\alpha}^{+}(F)}$ for all $\alpha \in(0,1)$. This proves $T(x) \in P=\bigcap_{\alpha \in(0,1)} \overline{P_{\alpha}^{+}\left(F^{\prime}\right)}$.

(2a) If for some $F \in I^{+}\left(Y^{\prime}\right)$ and some $\nu \in(0,1), T^{-n} G^{\prime}(\nu) \subset Y^{\prime}$ for all $n \geqq 0$ then $G_{0}(\nu)=G^{\prime}(\nu) \bigcap_{A} Y^{\prime}$ satisfies the condition $G_{0}(\nu) \cap A \gg A$. Since $T^{-1} G_{0}(\nu) \subset Y^{\prime}$ Lemma 3.7 implies $T^{-1} G_{0}(\nu) \cup A \gg A$. Thus the definition of $G_{1}(\nu)$ reduces to $G_{1}(\nu)=T^{-1} G_{0}(\nu)$. Repeating this we see that $G_{n}(\nu)=T^{-n} G_{0}(\nu) \subset Y^{\prime}$ and $G_{n}(\nu) \cup A \gg A$ for all $n \geqq 0$. Thus $G_{n+1}(\nu)=T^{-1} G_{n}(\nu)$ and hence $T^{-1} P_{\nu}^{+}(F) \subset P_{\nu}^{+}(F)$. Taking lim sup we get $T^{-1} P^{+}\left(F^{\prime}\right) \subset P^{+}(F)$. This and part (1) implies $P^{+}\left(F^{\prime}\right) \in I\left(Y^{\prime}\right)$. Define $G=P_{u}^{+}(F)$ and $F^{*}=\bar{G}$. It was shown in the proof of (1) that $G \cup$ $A \gg A$ and therefore $F^{*} \cup A \gg A$. Also from $T^{-1} G \subset G$ follows

$$
T^{-1} F^{*} \subset F^{*} \text {. }
$$

(2b) If $P^{+}(F) \subset$ int $_{\Sigma}, Y^{\prime}$ then $P^{+}\left(F^{\prime}\right) \cup A \subset\left(\right.$ int $\left._{\Sigma}, Y^{\prime}\right) \cup A=\operatorname{int}_{\Sigma} Y$. But $P^{+}(F) \cup A=\bigcap_{\alpha \in(0,1)}\left[P_{\alpha}^{+}(F) \cup A\right]$ and since $Y$ is compact there exists a $\nu \in(0,1)$ such that $P_{u}^{+}(F) \cup A \subset \operatorname{int}_{\Sigma} Y$, i.e., $F^{*}=P_{\alpha}^{+}(F) \subset$ int $_{\Sigma^{\prime}} Y^{\prime}$ thus proving the assertion.

(2c) If $Y$ is locally connected at each point of $F \cup A$ then each $G(\alpha)$ may be chosen to satisfy the additional condition, $G(\alpha) \gg A$. To see this, by the local connectedness assumption there is a connected open $U_{x} \subset Y, x \in U_{x}$ for each $x \in F \cup A$. Define

$$
G(1)=\bigcup_{x \in F \cup A} U_{x} \text {. }
$$


From properties (3.6), (3.2), and (3.1) we see that $U_{x} \cup F \cup A \gg F \cup A \gg$ $A$ and therefore $G(1)=\bigcup_{x \in F \cup A}\left(U_{x} \cup F \cup A\right) \gg A$. Assume $G(1 / n)$ has been defined and let $U \subset Y$ be open such that $A \subset U \subset \bar{U} \subset G(1 / n)$ and $d(A, Y-U)<1 /(n+1)$. Again for each $x \in F \cup A$ there is an open connected $U_{x} \subset Y, x \in U_{x} \subset Y$. Define $G(1 /(n+1))=\bigcup_{x \in F^{\cup} A} U_{x}$. Finally for $\alpha \in(1 /(n+1), 1 / n)$ define $G(\alpha)=G(1 / n)$.

Now $G^{\prime}(\alpha)=G(\alpha) \cap Y^{\prime}$ is open in $Y^{\prime}$ and $G^{\prime}(\alpha) \cup A=G(\alpha) \gg A$. Thus in the proof of (2a) $G_{0}(\nu)=G^{\prime}(\nu)$ and it follows that $G$ is open in $Y^{\prime}$. To see that $G \cup A$ is open in $Y$ let $Z=Y-G \cup A$. Since $G_{0}(\nu) \cup A=G^{\prime}(\nu) \cup A=G(\nu)$ is open in $Y$ then defining

$$
E=Y-\left[G_{0}(\nu) \cup A\right]
$$

we see that $E$ is closed $Y$ and $E \subset Y-A=Y^{\prime}$. Since $G_{0}(\alpha) \subset G, Z \subset$ $Y-G_{0}(\alpha) \cup A=E$ and also $Z=(Y-G) \cap(Y-A)=(Y-G) \cap Y^{\prime}=$ $Y^{\prime}-G$ implies $Z$ is closed in $Y^{\prime}$. This and $Z \subset E \subset Y^{\prime}$ implies $Z$ is closed in $Y$. This completes the proof.

REMARKs. (1) Clearly by replacing $T$ by $T^{-1}$ throughout we may construct $P^{-}(F)$ for $F \in I^{-}(Y)$ and a similar theorem holds.

(2) The empty set $\varnothing \in I^{+}\left(Y^{\prime}\right)$. However, $P^{+}(\varnothing)$ need not be empty. For example take $A=\{0\}$ the origin in $R^{n}$ and let $A$ be an asymptotically stable fixed point of a homeomorphism $T: R^{n} \rightarrow R^{n}$, i.e., $T^{l}(x) \rightarrow 0$ as $l \rightarrow \infty$. Let $\Sigma=\{\|x\| \leqq 2\}$ and $Y=\{\|x\| \leqq 1\}$. Then $P^{+}(\varnothing)=Y-A=Y^{\prime}$.

CoRollary 3.9. If for some $F \in I^{+}\left(Y^{\prime}\right), Y$ is locally connected at each point of $F \cup A$ and if there exists an $x \in \Sigma-Y$ such that $T^{n}(x) \rightarrow$ $F \cup A$ as $n \rightarrow \infty$, then $P^{+}(F) \cap \partial_{\Sigma} Y \neq \varnothing$.

Proof. If not then from part (2) of the theorem there exists $F^{*}=\bar{G} \in I^{-}\left(Y^{\prime}\right)$ where $G \cup A$ is open in $Y$. For $n$ sufficiently large then $z=T^{n}(x) \in G$. But then $x=T^{-n} z$ and $T^{-1} F^{*} \subset F^{*} \subset Y^{\prime}$ leads to a contradiction since $x \in \Sigma-Y=\Sigma^{\prime}-Y^{\prime}$.

Definition. Let $A, Y$, and $T$ be defined as before. Then $A$ is said to satisfy property $M$ in $Y$ with respect to $T$ if $I\left(Y^{\prime}\right)=\{\varnothing\}$. In particular if $A$ is connected and $T$ is a homeomorphism defined in a neighborhood of $A$ leaving $A$ invariant, $T A=A$, then $A$ satisfies property $M$ if and only if $A$ is maximal in the class of $T$-invariant continuua contained in $Y$, or stated more simply, $A$ is maximal in $Y$ with respect to $T$.

Lemma 3.10. If $A$ satisfies property $M$ and $E \in I^{+}\left(Y^{\prime}\right)$ then $\bigcap_{n=0}^{\infty} T^{n} E=\varnothing$. In particular for all $x \in E, d\left(T^{n}(x), A\right) \rightarrow 0$ an $n \rightarrow \infty$. 
$A$ similar statement holds for $E \in I^{-}(Y)$ with $T$ replaced by $T^{-1}$.

Proof. $\bigcap_{n=0}^{\infty} T^{n} E \in I\left(Y^{\prime}\right)$ follows from the fact that $T E \subset E, E \cup$ $A \gg A$, Lemma 3.7 and property (3.4.)

Another immediate result of Theorem 3.8 is

Corollary 3.11. If $A$ satisfies property $M$ then either $P^{+}(\varnothing)=$ $\varnothing$ or $P^{+}(\varnothing) \cap \partial_{\Xi} Y \neq \varnothing$.

THEOREM 3.12. Let $A$ be a fixed point of $T$ which is maximal in $Y$. If $Y$ is locally connected at $A$ then either $P^{+}(\varnothing) \cap \partial_{\Sigma} Y \neq \varnothing$ or $A$ is asymptotically stable under $T^{-1}$.

Proof. If $P^{+}(\varnothing) \cap \partial_{\Sigma} Y=\varnothing$ then from Theorem $3.18(2 \mathrm{~b})$ there exists an open $G \subset Y^{\prime}$ such that $\bar{G}=F^{*} \in I^{-}(Y)$ and $G \cup A$ is open in $Y$. Let $V \subset Y$ be an open neighborhood of $A$. We must find an open neighborhood $U$ of $A$ such that $T^{-n} U \subset V$ for all $n \geqq 0$ and $d\left(T^{-n} U, A\right) \rightarrow 0$ as $n \rightarrow \infty$. From Corollary $3.10 \bigcap_{n=0}^{\infty} T^{-n}(G \cup A)=A$ and therefore there is an $n_{0}$ such that $T^{-n_{0}}(G \cup A)=\bigcap_{n=0}^{n_{0}} T^{-n}(G \cup$ $A) \subset V$. Simply define $U=T^{-n_{0}}(G \cup A)$ and the theorem is proved.

COROLLARY 3.13. Under the condition of Theorem 3.12 if there exists an $x \in \Sigma$ such that $d\left(T^{n} x, A\right) \rightarrow 0$ as $n \rightarrow \infty$ then there exists a continuum of such points extending from $A$ to $\partial_{\Sigma} Y$.

If the "maximal" assumption is dropped, Corollary 3.13 is no longer true as illustrated by the following

ExAmple. We construct a mapping $T: R^{2} \rightarrow R^{2}$ such that the origin is fixed and $A^{+}=\left\{p \in R^{2}-(0,0): T^{n} p \rightarrow(0,0)\right.$ as $\left.n \rightarrow \infty\right\}$ is discrect. Define $f:[0, \infty) \rightarrow[0,1]$ to be continuous, $f\left(2^{-j}\right)=1 /(j+1)$ for $j \in Z^{+}, f$ is linear on each interval $\left[2^{-j-1}, 2^{-j}\right]$ and $f(t)=1$ for $t \geqq 1$. Clearly $f$ is monotone increasing. Define $E=\left\{(x, y) \in R^{2}: x=\right.$ 0 or $\left.2^{-n}, n \in Z^{+}\right\}$and let $\delta: R^{2} \rightarrow R$ be the distance from $E$, i.e., $\delta(x, y)=$ $d((x, y), E)$. Finally define $T$ by $T(x, y)=\left(x_{1}, y_{1}\right)$ where

$$
\begin{aligned}
& y_{1}=y-f(\delta(x, y)) \\
& x_{1}=\frac{1}{2} x
\end{aligned}
$$

The $y$ - axis consists of fixed points and $A^{+}=\left\{\left(2^{j}, 0\right): j \in Z\right\}$. To see this note that for points $(x, y) \in E, T^{n}(x, y) \rightarrow(0, y)$ as $n \rightarrow \infty$ and only those in $A^{+}$approach $(0,0)$. Further, for $\left(x_{0}, y_{0}\right) \notin E$ we will show that $T^{n}\left(x_{0}, y_{0}\right)$ becomes unbounded. For $n$ sufficiently large $T^{k}\left(x_{0}, y_{0}\right)$ is in 
the strip $|x|<1$ for all $k \geqq n$ so we might as well assume $\left|x_{0}\right|<1$ to start with. Then $\delta\left(x_{0}, y_{0}\right)=\delta_{0}$ where $2^{-l} \leqq \delta_{0}<2^{-l+1}$ for some integer $l \geqq 1$ and if $\left(x_{n}, y_{n}\right)=T^{n}\left(x_{0}, y_{0}\right), \delta\left(x_{n}, y_{n}\right)=2^{-n} \delta_{0} \geqq 2^{-n-l}$. Therefore, $f\left(\delta\left(x_{n}, y_{n}\right)\right) \geqq f\left(2^{-n-l}\right)=1 /(n+l+1)$. But $y_{N}=y_{0}-\sum_{n=0}^{N-1} f\left(\delta\left(x_{n}\right.\right.$, $\left.\left.y_{n}\right)\right) \leqq y_{0}-\sum_{n=0}^{N-1} 1 /(n+l+1)$ shows that $y_{N} \rightarrow-\infty$ as $N \rightarrow \infty$.

If we take $\Sigma=R^{2}$ and $Y$ the unit disc $\Delta=\left\{(x, y) \in R^{2}: x^{2}+y^{2} \leqq\right.$ $1\}$ and $A=(0,0)$ we see that $I^{+}\left(Y^{\prime}\right)=I\left(Y^{\prime}\right)$ and $F \in I^{+}\left(Y^{\prime}\right)$ implies $F=\{(0, y): \alpha \leqq y \leqq \beta, y \neq 0\}$ for some $\alpha$ and $\beta,-1 \leqq \alpha \leqq 0 \leqq \beta \leqq 1$. For all $F \in I^{+}\left(Y^{\prime}\right), P^{+}(F)=\{(0, y): 0 \leqq y \leqq 1\} \cup F$. Of course $A$ fails to be maximal.

Taking $\Sigma=E, Y=\Delta \cap E$ and $A=(0,0)$ we see that $I^{+}\left(Y^{\prime}\right)$ is the same but $P^{+}\left(F^{\prime}\right)=F$ for all $F \in I^{+}\left(Y^{\prime}\right)$. Thus the assumption of local connectedness in Corollary 3.9 cannot be dropped.

4. Flows. We now consider the behavior of a flow $\pi: X \times R \rightarrow$ $X$ near a compact invariant subset $I \subset X$ a metric space. Suppose $K$ is a compact neighborhood of $I$ and define

$$
A^{+}=\left\{x \in K: x \cdot R^{+} \subset K \text { and } \omega(x) \subset I\right\},
$$

the stable set associated with $I$.

Definition. We say $I$ is maximal in $K$ with respect to $\pi$ if whenever $F \subset K$ is closed, $\pi$-invariant and $F \gg I$ then $F=I$. In particular if $I$ is connected then $I$ is maximal if it is a maximal element in the class of closed $\pi$-invariant continua contained in $K$.

Lemma 4.1. If $I$ is maximal in $K$ then $A^{+}$is closed.

Proof. Let $x_{n} \in A^{+}, x_{n} \rightarrow x$. Then clearly $x \cdot R^{+} \subset K$ and hence $\omega(x)$ is compact and therefore connected. There exists a subsequence, again call it $x_{n}$, and sequences $t_{n}$ and $\tau_{n}$ in $R^{+}, t_{n}<\tau_{n}$ such that $\overline{\lim }_{n \rightarrow \infty} x_{n} \cdot t_{n}=\omega(x)$ and $\overline{\lim }_{n \rightarrow \infty} x_{n} \cdot \tau_{n}=I^{\prime} \subset I$ for some nonempty subset $I^{\prime}$ of $I$ (this follows directly from the continuity of the flow). Define $F=\varlimsup_{n \rightarrow \infty} x_{n} \cdot\left[t_{n}, \tau_{n}\right]$. Then $F$ is clearly closed and since $\omega(x)$ is connected so is $F$ and $F \supset \omega(x) \cup I^{\prime}$. Defining $F^{\prime}=F \cup I$, let us show that $F^{\prime}$ is invariant. If suffices to show $F^{\prime}-\omega(x) \cup I$ is invariant. Let $y \in F^{\prime}-\omega(x) \cup I$. Then there is a sequence $s_{n} \in\left(t_{n}, \tau_{n}\right)$ such that $x_{n} \cdot s_{n} \rightarrow y$. Since $I$ and $\omega(x)$ are invariant, $s_{n}-t_{n} \rightarrow \infty$ and $\tau_{n}$ $s_{n} \rightarrow \infty$ with $n$. Now let $T>0$ and let $N$ be so large that $\tau_{n}-s_{n}>$ $T$ for all $n \geqq N$. Then for $n \geqq N, x_{n} \cdot\left(s_{n}+T\right) \in x_{n} \cdot\left[t_{n}, \tau_{n}\right]$ and therefore $y \cdot T=\lim x_{n} \cdot\left(s_{n}+T\right) \in F^{\prime}-\omega(x) \cup I$. Similarly for $T<0$. Therefore, $F^{\prime}-\omega(x) \cup I$ and hence $F^{\prime}$ is invariant. Clearly $F^{\prime}$ is closed and since $F$ is connected and $F \cup I \supset I^{\prime} \neq \varnothing$ it follows from (3.6) that $F^{\prime} \gg I$, i.e., $F \cup \gg I$. But $I$ maximal implies $F \subset I$ and there- 
fore $\omega(x) \subset I$ completing the proof.

It is easily seen that if we define $F=\bigcap_{t \in R^{+}} A^{+} \cdot t$ then $F$ is invariant and $F \gg I$. Thus if $I$ is maximal in the neighborhood $K$ we have $F=I$, i.e., $A^{+}$satisfies

Condition B. A subset $D, I \subset D \subset K$, satisfies Condition B if $\bigcap_{t \in R^{+}} D \cdot t=I$. Consider also

Condition $\mathrm{B}^{\prime}$. A subset $D, I \subset D \subset K$, satisfies Condition $\mathrm{B}^{\prime}$ if for all $x \in D-I$ there exists $f(x) \in R^{-}$such that $x \cdot f(x) \in X-K$.

Definition. A subset $D \subset K$ is relatively negatively invariant (relative to $K$ ) if for all $x \in D$ and $t \in R^{-}$we have $[t, 0] \cdot x \subset D$ whenever $[t, 0] \cdot x \subset K$. The set $A^{+}$is an example of such a subset.

Lemma 4.2. Consider a subset $D, I \subset D \subset K$.

(1) If $D$ is positively invariant and satisfies Condition $\mathrm{B}^{\prime}$ then it satisfies Condition B.

(2) If $D$ is relatively negatively invariant and satisfies Condition $\mathrm{B}$ then it satisfies Condition $\mathrm{B}^{\prime}$.

Proof. (1) If not, there exists $x \in \bigcap_{t \in R^{+}} D \cdot t-I$. If $\tau>0$ then $x \in D \cdot \tau$ implies $x \cdot[-\tau, 0] \subset D \cdot \tau \cdot[-\tau, 0]=\bigcup_{\sigma \in[-\tau, 0]} D \cdot(\tau+\sigma) \subset D$ since $\tau+\sigma \geqq 0$ and $D$ is positively invariant. Thus for all $\tau>0$, $x \cdot[-\tau, 0] \subset D$ contradicting Condition $\mathrm{B}^{\prime}$.

(2) If not, there is an $x \in D-I$ such that $x \cdot R^{-} \subset K$ and since $D$ is relatively negatively invariant, $x \cdot R^{-} \subset D$. Thus for all $t \geqq 0$ there is a $y \in D-I$ such that $y \cdot t=x$, namely $y=x \cdot(-t)$. But then $x \in D \cdot t$ for all $t \geqq 0$ and therefore $x \in \bigcap_{t \in R^{+}} D \cdot t$ contradicting Condition B.

Lemma 4.3. Let $D^{+} \subset K$ be a closed, positively invariant subset which satisfies Condition B. Then (1) there exists an open neighborhood $V$ of $I$ such that $V \subset K$ and for all $x \in V \cap D^{+}, x \cdot R^{+} \cap \partial_{X} K=\varnothing$ and (2) for any open neighborhood $V$ of $I$ with $V \subset K$, there exists a $t_{0}>0$ such that $D^{+} \cdot\left[t_{0}, \infty\right) \subset V$.

Proof. (2) This follows directly from Condition B. (1) If not, there exist $x_{n} \in D^{+}, t_{n} \in R^{+}$such that $x_{n} \rightarrow I$ and $x_{n} \cdot t_{n} \in D^{+} \cap \partial_{X} K$. Then $t_{n} \rightarrow \infty$ since $I$ is invariant. Let $y_{n}=x_{n} \cdot t_{n}$. By compactness there is a subsequence, again call it $y_{n}$, such that $y_{n} \rightarrow y \in D^{+} \cap \partial_{X} K$. Now $y_{n} \cdot\left[-t_{n}, 0\right] \subset D^{+}$implies that $y \cdot R^{-} \subset D^{+}$. Thus since $D^{+}$is also positively invariant, $y \cdot R \subset D^{+}$. But $y \cdot R$ is invariant which contradicts $\cap D^{+} \cdot t=I$ and completes the proof. 
5. Flow near an invariant Jordan curve. In this section we consider an $n$-dimensional manifold $M^{n}$ and a flow $\pi: M^{n} \times R \rightarrow M^{n}$ which admits a Jordan curve $J$ as an invariant set. Assume $X$ is a closed neighborhood of $J$ which is a solid torus having $J$ as "center line", i.e., there exists a homeomorphism $h: V^{n-1} \times S^{1} \rightarrow X$ such that $h\left(\{0\} \times S^{1}\right)=J$ where $V^{n-1}=\left\{x \in R^{n-1}:\|x\| \leqq 1\right\}$ is the closed unit $n-$ 1 ball and $S^{1}=\{Z \in C:|Z|=1\}$. Henceforth, by an open (closed) tubular neighborhood of $J$ we will mean the image $h\left(V_{r} \times S^{1}\right)$ where $V_{r} \subset R^{n-1}$ is the open (closed) $n-1$ ball of radius $r, 0<r \leqq 1$.

Define $A^{+}=\left\{x \in X: x \cdot R^{+} \subset X, \omega(x) \subset J\right\}$ and assume there exists $D^{+} \subset A^{+}$which is a closed, positively invariant and relatively negatively invariant subset of $X$ satisfying Condition $B$ (and hence also Condition $\mathrm{B}^{\prime}$ by Lemma 4.2). If $J$ is maximal in $X$ we may simply choose $D^{+}=A^{+}$. By Lemma 4.3 we may choose an open tubular neighborhood $V$ of $J$ such that for all $x \in \bar{V} \cap D^{+}, x \cdot R^{+} \cap \partial X=\varnothing$. Under an additional condition (Condition A) we will prove that ( $X$ $V) \cap D^{+}$carries a Čech 1 -cycle $Z$ which is homologous over $D^{+}$to the basic 1-cycle carried by $J$. In the case in which $X$ is convex to the flow [6] we may take $D^{+}=A^{+}$and apply the results repeatedly using a sequence $V_{n} \subset V_{n+1}$ such that $\cup V_{n}=\operatorname{int} X$. Then by applying a continuity argument to the nested compact subsets $K_{n}=\left(X-V_{n}\right) \cap$ $A^{+}$we obtain a 1-cycle on $\cap K_{n}=A^{+} \cap \partial X$ which agrees with the result of [3] in which $J$ is a periodic orbit of a continuous vector field in $R^{3}$ and in addition $J$ is assumed to be isolated as an invariant set.

We now describe Condition $\mathrm{A}$ and the results. Let $E=V^{n-1} \times$ $R$ be the covering space of $V^{n-1} \times S^{1}$ with projection $p^{\prime}: E \rightarrow V^{n-1} \times S^{1}$ defined by $p^{\prime}(y, t)=\left(y, e^{2 \pi i t}\right)$. Then $E$ covers $X$ with projection $p=$ $h \circ p^{\prime}$. Let $q: E \rightarrow R$ be projection onto the second factor, $(x, t) \rightarrow t$. Since $p$ is a local homeomorphism we have the local covering flow $\tilde{\pi}$ in $E$ defined as follows: Let $U$ be a neighborhood in $E$ such that $(p \mid U): U \rightarrow p(U) \subset X$ is a homeomorphism. For $(y, t) \in U \times R$ such that $\pi(p(y),[0, t]) \subset p(U)$ define $\tilde{\pi}(y, t)=(p \mid U)^{-1} \pi(p(y), t) \in U$. One can easily verify that $\tilde{\pi}$ is continuous for all $(y, t)$ such that $\pi(p(y)$, $[0, t]) \subset \operatorname{int} X$.

Define functions $t^{+}, t^{-}: D^{+}-(J \cup \partial X) \rightarrow R^{-}$as follows:

$$
\begin{aligned}
& t^{-}(x)=\inf \left\{t<0: x \cdot[t, 0] \subset D^{+} \cap \operatorname{int} X\right\} \\
& t^{+}(x)=\sup \left\{t<0: x \cdot\left(t^{-}(x), t\right) \subset D^{+} \cap \operatorname{int}(X-V)\right\} .
\end{aligned}
$$

Since $D^{+}$satisfies Condition $\mathrm{B}^{\prime}$, for any $x \in D^{+}-(J \cup \partial X)$ the point $x \cdot t$ must leave $X$ for some $t<0$ and therefore $t^{-}(x)>-\infty$ and $x \cdot t^{-}(x) \in \partial X$. Also it is clear that $t^{-}(x)<t^{+}(x)$.

Lemma 5.1. If $V$ is chosen as above then $t^{-}$is upper-semicon- 
tinuous and $t^{+}$is lower-semicontinuous on $D^{+}-(J \cup \partial X)$. Further there exists an $M>0$ such that $t^{+}(x)+t^{-}(x)<M$ for all $x \in D^{+}-$ $(J \cup \partial X)$.

Proof. Let $\varepsilon>0$ and $\tau=t^{-}(x)+\varepsilon$. Then $x \cdot[\tau, 0] \subset \operatorname{int} X$ and by continuity there is a neighborhood $N$ of $x$ such that for $\xi \in N$, $\xi \cdot[\tau, 0] \subset \operatorname{int} X$. Thus $t^{-}(\xi) \leqq \tau$ for all $\xi \in N$, i.e., $t^{-}$is upper-semi-continuous at $x$. Now let $\varepsilon>0$ be such that $t^{-}(x)<t^{+}(x)-\varepsilon<t^{+}(x)$. From the upper-semicontinuity of $t^{-}$choose a neighborhood $N$ of $x$ such that $t^{-}(\xi)<t^{+}(x)-\varepsilon$ for $\xi \in N$. Let $\tau=t^{+}(x)-\varepsilon$. Then $x \cdot\left(t^{-}(x)\right.$, $\tau] \subset D^{+} \cap \operatorname{int}(X-V)$. We claim there exists a neighborhood $N^{\prime} \subset N$ of $x$ such that $\xi \in N^{\prime}$ implies $\xi \cdot\left(t^{-}(\xi), \tau\right] \subset D^{+} \cap \operatorname{int}(X-V)$. Granting this for the moment we then have $t^{+}(\xi) \geqq \tau$ for $\xi \in N^{\prime}$, i.e., $t^{+}$is lower-semicontinuous at $x$. To prove the claim, from the given condition on $V$ there is an open $U$ such that $\bar{V} \subset U \subset \bar{U} \subset$ int $X$ and for all $y \in \bar{V} \cap D^{+}, y \cdot R^{+} \subset U$. Hence if $y \in D^{+} \cap \operatorname{int}(X-U)$ we have $y \cdot\left(t^{-}(y), 0\right] \subset D^{+} \cap(X-V)$. Now choose $s \in\left(t^{-}(x), \tau\right)$ such that

$$
x \cdot\left(t^{-}(x), s\right] \subset D^{+} \cap \operatorname{int}(X-U)
$$

and a neighborhood $N^{\prime} \subset N$ such that $\xi \cdot s \subset D^{+} \cap \operatorname{int}(X-U)$ and $\xi \cdot[s, \tau] \subset D^{+} \cap \operatorname{int}(X-V)$ for all $\xi \in N^{\prime}$. Applying the previous statement to $y=\xi \cdot s$ we get

$$
\xi \cdot s \cdot\left(t^{-}(\xi \cdot s), 0\right]=\xi \cdot\left(t^{-}(\xi), s\right] \subset D^{+} \cap \operatorname{int}(X-V) .
$$

Combining the inclusions in the last two sentences we obtain the claim. To prove the last assertion of the lemma we argue negatively and assume there exists a sequence $x_{n} \in D^{+}-(J \cup \partial X)$ and times $t_{n}$, $s_{n}$ such that $t^{-}\left(x_{n}\right)<t_{n}<s_{n}<t^{+}\left(x_{n}\right)$ where $\sigma_{n}=s_{n}-t_{n} \rightarrow \infty$. But then $x_{n} \cdot\left[t_{n}, s_{n}\right] \subset D^{+} \cap(X-V)$ and therefore there exist points $y_{n}=$ $x_{n} \cdot t_{n} \in D^{+} \cap(X-V)$ such that $y_{n} \cdot\left[0, \sigma_{n}\right] \subset D^{+} \cap(X-V)$. From compactness of the latter there exists $y \in \overline{\lim } y_{n}$ such that $y \cdot R^{+} \subset$ $D^{+} \cap(X-V)$ contradicting Condition B and the lemma is proved. Define

$$
\Delta=p^{-1}\left(D^{+}\right) \subset E, \widetilde{J}=p^{-1}(J)=\{0\} \times R \subset E, \tilde{J}=\Delta-\left(\widetilde{J} \cup p^{-1}(\partial X)\right)
$$

and the lifted functions $T^{+}, T^{-}: \widetilde{\Delta} \rightarrow R^{-}$by $T^{ \pm}(y)=t^{ \pm} \circ p(y)$. Assume that our dynamical system satisfies

Condition A. There exists a bounded connected subset $\Gamma \subset \tilde{J}$ and a sequence $y_{n} \in \Gamma$ such that $q\left(y_{n} \cdot T^{-}\left(y_{n}\right)\right) \rightarrow-\infty$.

REMaRks. (1) We do not exclude the possibiliy that some trajectories in $A^{+}$could be asymptotic as $t \rightarrow \infty$ to critical points on $J$. 
(2) We do not require that $J$ admits a local (quasi) section. However, if it does admit one of the form $\Sigma=p\left(V^{n-1} \times\{0\}\right)$ and if $A^{+}-J \neq \varnothing$ and $J$ is assumed maximal in $X$, then applying Corollary 3.9 we may choose $\Gamma$ to be any component of $p^{-1}\left(P^{+}(\phi)-\partial X\right)$ such that $\overline{p(T)}$ contains the singleton $A=\Sigma \cap J$. Condition $\mathrm{A}$ is then satisfied by choosing any sequence $y_{n} \in \Gamma$ such that $p\left(y_{n}\right) \rightarrow A$.

THEOREM 5.2. Let $V$ be an open tubular neighborhood of $J$ such that for all $x \in \bar{V} \cap D^{+}$we have $x \cdot R^{+} \cap \partial X=\varnothing$ and suppose that Condition A holds. Then $(X-V) \cap D^{+}$carries a Čech 1-cycle which is homologous over $D^{+}$to the basic 1-cycle carried by $J$. Here the coefficient group $G$ is taken to be either the reals $R$, the rationals $Q$ or the reals $\bmod 1, R_{1}$.

Proof. Define $K=(X-V) \cap D^{+}$. Among all open covers $\alpha$ of $X$ we consider only those which are finite and have the property that if $U_{1}, \cdots, U_{l} \in \alpha$ are such that $U_{\imath} \cap K \neq \varnothing, i=1, \cdots, l$, then $\bigcap_{i=1}^{l} U_{i}$ is nonempty if and only if $\bigcap_{i=1}^{l} U_{\imath} \cap K \neq \varnothing$. By [11] and the compactness of $X$ these covers form a cofinal subcollection of the collection of all open covers.

Let $\alpha$ be an open cover of $X$ and denote by $X_{\alpha}$ the nerve of $\alpha$ and by $K_{\alpha}$ the nerve of the induced cover on the closed subset $K \subset X$. Assume $\alpha$ is so fine that $H_{1}\left(X_{\beta}\right) \cong G$ for all covers $\beta>\alpha$, i.e., which refine $\alpha$. Define $Z=D^{+}-(J \cup \partial X)$ and consider the functions $t^{+}, t^{-}: Z \rightarrow R^{-}$defined earlier. Since $t^{+}$and $t^{-}$are respectively lower and upper-semicontinuous on $Z$, a normal, countably paracompact space and $t^{-}(x)<t^{+}(x)$ for all $x \in Z$ we have (Dowker [5]) a continuous function $\varphi: Z \rightarrow R^{-}$such that $t^{-}(x)<\varphi(x)<t^{+}(x)$ for all $x \in Z$. Clearly then $x \cdot \varphi(x) \in K$ for all $x \in Z$. Let $Y=p^{-1}(Z)$ and define $\Psi: Y \rightarrow R^{-}$by $\Psi(y)=\varphi \circ p(y)$. By Condition A there is a bounded connected subset $\Gamma \subset Y$ and a sequence $y_{n} \in \Gamma$ such that $q\left(y_{n} \cdot T^{-}\left(y_{n}\right)\right) \rightarrow-\infty$. From the second assertion of Lemma 5.1 we see that $0<\Psi\left(y_{n}\right)-T^{-}\left(y_{n}\right)<M$ and therefore $q\left(y_{n} \cdot \Psi\left(y_{n}\right)\right) \rightarrow-\infty$. Thus if we define the function $\lambda: \Gamma \rightarrow Y$ by $y \rightarrow y \cdot \Psi(y)$ we see that $\lambda$ is continuous and letting $C=\lambda(\Gamma)$ we see that $C$ is connected, $C \subset p^{-1}(K)$ and $\bar{C}$ is noncompact. Define $K^{\prime}=\overline{p(C)} \subset K$. Then $K^{\prime}$ is compact and connected.

Now let $\beta>\alpha$ be an open cover of $X$. We claim that there exists a nontrivial class $\gamma \in H_{1}\left(K_{\beta}\right)$ such that $\gamma$ is not in the kernel of the inclusion induced map $i_{*}: H_{1}\left(K_{\beta}\right) \rightarrow H_{1}\left(X_{\beta}\right)$. To prove this define $U_{0}=\bigcup\left\{U \in \beta: U \cap K^{\prime} \neq \varnothing\right\}$. It suffices to show the existence of a map $f: S^{1} \rightarrow U_{0}$ which is not null-homotopic over $X$. For letting $j: U_{0} \rightarrow X$ be inclusion, then $j \circ f$ is homotopic over $X$ to a map $\rho: S^{1} \rightarrow J$ of degree $n \neq 0$. This in turn implies that the subset $\operatorname{Im}(j \circ f) \subset X$ 
carries a cycle of $Z_{1}\left(X_{\beta}\right)$ generating a nontrivial class in $H_{1}\left(X_{\beta}\right)$. But $\operatorname{Im}(f) \subset U_{0}$ and by our choice of covers, any cycle on $X_{\beta}$ having vertices $U \in \beta$ such that $U \cap K \neq \varnothing$ is actually a cycle on $K_{\beta}$ which establishes the claim.

To verify the sufficient condition let $\widetilde{U} \subset U_{0}$ be the component containing $K^{\prime}$ and arguing negatively assume every map $f: S^{1} \rightarrow \widetilde{U}$ is null-homotopic. Then every closed curve in $\widetilde{U}$ can be lifted to a closed curve in the covering space $E$. But then (Spanier [10]) $p^{-1}(\widetilde{U})$ is a disjoint union of subsets each homeomorphic to $\widetilde{U}$ via $p$, i.e., there is a homeomorphism $g: \widetilde{U} \rightarrow W \subset E$ where $W$ is the component of $p^{-1}(\widetilde{U})$ containing $C$. Since $K^{\prime} \subset \widetilde{U}$ is compact, $g\left(K^{\prime}\right)$ is a compact subset of $E$. But $C \subset g\left(K^{\prime}\right)$ contradicting the fact that $\bar{C}$ is noncompact. Thus the claim is proved.

Since the coefficient group $G$ is divisible and $H_{1}\left(X_{\beta}\right) \cong G$ we see that the sequence $H_{1}\left(K_{\beta}\right) \stackrel{i^{*}}{\longrightarrow} H_{1}\left(X_{\beta}\right) \rightarrow 0$ is exact. Now form the Čech homology groups $H_{1}$ by taking: inverse limits along the cofinal sequence of open covers described earlier. Since all groups involved are either finite dimensional vector spaces or compact topological groups, and the inverse limit of exact sequences of such groups is itself exact, we see that the following sequence of Čech groups is exact:

$$
H_{1}(K) \longrightarrow H_{1}(X) \longrightarrow 0 \text {. }
$$

The flow induced map $h_{t}: D^{+} \rightarrow D^{+} \cdot t$ given by $h_{t}(x)=x \cdot t$ is a homeomorphism and from Condition $\mathrm{B}, \bigcap_{t \geqq 0} D^{+} \cdot t=J$. Thus, taking the inverse limit as $t \rightarrow \infty$ in the sequence

$$
H_{1}\left(D^{+} \cdot s, J\right) \stackrel{\left(h_{s-t}\right) *}{\longleftarrow} H_{1}\left(D^{+} \cdot t, J\right) \longleftarrow \cdots \longleftarrow \lim _{\longleftarrow} H_{1}\left(D^{+} \cdot t, J\right)
$$

and using the continuity of the Čech theory we obtain

$$
H_{1}\left(D^{+}, J\right) \cong \lim _{\leftarrow} H_{1}\left(D^{+} \cdot t, J\right) \cong H_{1}(J, J)=0 .
$$

The commutative diagram (all maps inclusion induced)

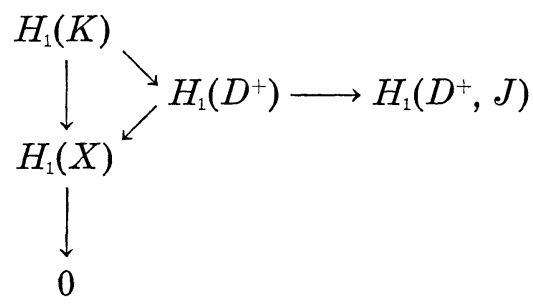

with exact column and $H_{1}(X) \cong G$ shows that we have constructed a Čech 1-cycle on $K$ which does not bound in $X$ but when considered 
as a cycle on $D^{+}$, it is homologous over $D^{+}$to the basic cycle carried by $J$. This completes the proof of the theorem.

\section{REFERENCES}

1. N. P. Bhatia and G. P. Szegö, Stability Theory of Dynamical Systems, Springer Grundlehren, Series Band 161, 1970.

2. Richard C. Churchill, Invariant sets which carry cohomology, JDE, 3 (1973), 523-550.

3. C. C. Conley, Invariant sets which carry a 1-form, J. Differential Equations, 8 (1970), 587-594.

4. C. C. Conley and R. Easton, Isolated invariant sets and isolating blocks, Trans. Amer. Math. Soc., 158 (1971), 35-61.

5. C. H. Dowker, Countably paracompact spaces, Canadian Math. J., 3 (1951), 219-224, Theorem 4.

6. Robert W. Easton, On the existence of invariant sets inside a submanifold convex to a flow, J. Differential Equations, 7 (1970), 54-68.

7. O. Hajek, Sections of dynamical systems in $E^{2}$, Czechoslovak Math. J., 15 (90)(1965), 205-211.

8. R. J. Sacker, A perturbation theorem for invariant manifolds and Hölder continuity, J. Math. and Mechanics, 18 (1969), 705-762, Theorem 8.2.

9. ㄴ. Asymptotic approach to periodic orbits, Technical report 1972/9, Instituto Math. Ulisse Dini, Universita Degli Studi di Firenze, 1972.

10. E. H. Spanier, Algebraic Topology, McGraw-Hill, 1966, Lemma 9, p. 78.

11. R. L. Wilder, Topology of Manifolds, Amer. Math. Soc. Colloq. Publ., Vol. 32, Lemma 8.9, p. 135.

Received November 30, 1972. This research was supported by U. S. Army Grant DA-ARO-D-31-124-71-G176 at the University of Southern California.

UNIVERSITY OF SOUTHERN CALIFORNIA 



\section{PACIFIC JOURNAL OF MATHEMATICS}

\section{EDITORS}

RICHARD ARENS (Managing Editor)

University of California

Los Angeles, California 90024

R. A. BeAumont

University of Washington

Seattle, Washington 98105
J. DugundJI*

Department of Mathematics

University of Southern California

Los Angeles, California 90007

D. Gilbarg and J. Milgram

Stanford University

Stanford, California 94305

\section{ASSOCIATE EDITORS}

E. F. BECKENBACH

B. H. NEUMANN

F. WOLF

K. YoSHIDA

\section{SUPPORTING INSTITUTIONS}

UNIVERSITY OF BRITISH COLUMBIA
CALIFORNIA INSTITUTE OF TECHNOLOGY
UNIVERSITY OF CALIFORNIA
MONTANA STATE UNIVERSITY
UNIVERSITY OF NEVADA
NEW MEXICO STATE UNIVERSITY
OREGON STATE UNIVERSITY
UNIVERSITY OF OREGON
OSAKA UNIVERSITY

UNIVERSITY OF BRITISH COLUMBIA CALIFORNIA INSTITUTE OF TECHNOLOGY UNIVERSITY OF CALIFORNIA MONTANA STATE UNIVERSITY NEW MEXICO STATE UNIVERSITY UNIVERSITY OF OREGON OSAKA UNIVERSITY
UNIVERSITY OF SOUTHERN CALIFORNIA STANFORD UNIVERSITY UNIVERSITY OF TOKYO UNIVERSITY OF UTAH WASHINGTON STATE UNIVERSITY UNIVERSITY OF WASHINGTON AMERICAN MATHEMATICAL SOCIETY NAVAL WEAPONS CENTER

* C. R. DePrima California Institute of Technology, Pasadena, CA 91109, will replace J. Dugundji until August 1974. 


\section{Pacific Journal of Mathematics}

\section{Vol. 51, No. $1 \quad$ November, 1974}

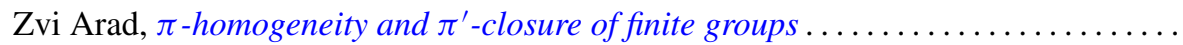

Ivan Baggs, A connected Hausdorff space which is not contained in a maximal

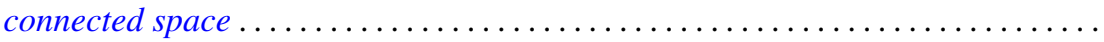

Eric Bedford, The Dirichlet problem for some overdetermined systems on the unit ball in $C^{n}$

R. H. Bing, Woodrow Wilson Bledsoe and R. Daniel Mauldin, Sets generated by

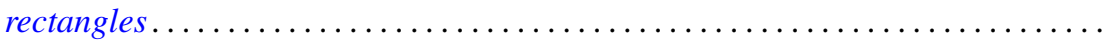

Carlo Cecchini and Alessandro Figà-Talamanca, Projections of uniqueness for

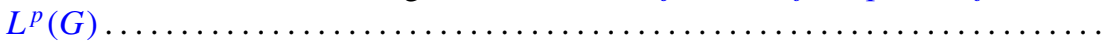

Gokulananda Das and Ram N. Mohapatra, The non absolute Nörlund summability of Fourier series .

Frank Rimi DeMeyer, On separable polynomials over a commutative ring ........ Richard Detmer, Sets which are tame in arcs in $E^{3} \ldots \ldots \ldots \ldots \ldots \ldots \ldots \ldots$

William Erb Dietrich, Ideals in convolution algebras on Abelian groups ..........

Bryce L. Elkins, A Galois theory for linear topological rings .................

William Alan Feldman, A characterization of the topology of compact convergence on $C(X)$.

Hillel Halkin Gershenson, A problem in compact Lie groups and framed cobordism

Samuel R. Gordon, Associators in simple algebras.

Marvin J. Greenberg, Strictly local solutions of Diophantine equations

Jon Craig Helton, Product integrals and inverses in normed rings . . . . . . . . . . . .

Domingo Antonio Herrero, Inner functions under uniform topology . . .

Jerry Alan Johnson, Lipschitz spaces .

Marvin Stanford Keener, Oscillatory solutions and multi-point boundary value

functions for certain nth-order linear ordinary differential equations.

John Cronan Kieffer, A simple proof of the Moy-Perez generalization of the

Shannon-McMillan theorem .......................

Joong Ho Kim, Power invariant rings

Gangaram S. Ladde and V. Lakshmikantham, On flow-invariant sets .

Roger T. Lewis, Oscillation and nonoscillation criteria for some self-adjoint even

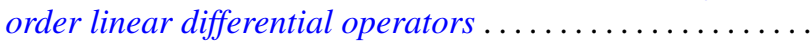

Jürg Thomas Marti, On the existence of support points of solid convex sets ..

John Rowlay Martin, Determining knot types from diagrams of knots . .

James Jerome Metzger, Local ideals in a topological algebra of entire functions

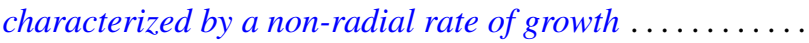

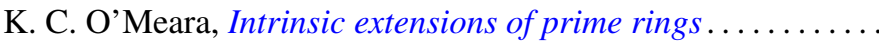

Stanley Poreda, A note on the continuity of best polynomial approximations ..

Robert John Sacker, Asymptotic approach to periodic orbits and local prolongations

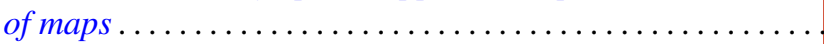

Eric Peter Smith, The Garabedian function of an arbitrary compact set . .

Arne Stray, Pointwise bounded approximation by functions satisfying a side condition

John St. Clair Werth, Jr., Maximal pure subgroups of torsion complete abelian

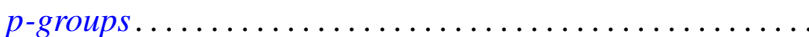

